\title{
Overview of the chapters
}

\section{1: Laboratory experimentation in marketing}

Marketing academics, managers, public policy makers, and litigators often ponder questions that involve relationships between alternative treatments or strategies and people's responses. Among the variety of research approaches available to them, only experimental designs afford strong causal inferences about such relationships. The chapter reviews the nature of such experiments, discusses the role of laboratory versus field experiments and explores the design of lab experiments along various dimensions.

\section{2: Field experiments}

In a digitally enabled world, experimentation is easier. This chapter explores what this means for marketing researchers, and the subtleties of designing field experiments for research. It gives guidelines for interpretation and describes the potential advantages and disadvantages of this methodology for classic areas of marketing.

\section{3: Conjoint Analysis}

This chapter offers an overview of Conjoint Analysis, with an eye toward implementation and practical issues. After reviewing the basic assumptions of Conjoint Analysis, I discuss issues related to implementation; data analysis and interpretation; and issues related to ecological validity. In particular, I discuss recent evidence regarding consumers' attention in Conjoint Analysis surveys, how it may be increased and modeled, and whether responses in Conjoint Analysis surveys are predictive of real-life behavior. Each section concludes with practical recommendations.

\section{4: Time-series models of short-run and long-run marketing impact}

Determining the long-term impact of marketing actions is strategically important, yet more challenging than uncovering short-term results. This chapter describes persistence modeling on time-series data as a promising method for long-term impact detection, especially as longitudinal databases in marketing are becoming more prevalent. We provide a brief technical introduction to each step in persistence modeling, along with a set of illustrative marketing studies that have used such models. Next, we summarize various marketing insights that have been derived from the use of persistence models in marketing. 


\section{5: Panel data methods in marketing research}

We review panel data models popular in marketing applications and highlight some issues, potential solutions, and trade-offs that arise in their estimation. Panel data studies controlling for unobservables often show dramatically different estimates than cross-sectional studies. We focus on models with unobservable individual-specific effects and address some misconceptions appearing in marketing applications.

\section{6: Causal inference in marketing applications}

This chapter summarizes the major methods of causal inference and comments on the applicability of these methods to marketing problems.

\section{7: Modeling choice processes in marketing}

This chapter examines the use of choice models in marketing. After briefly describing the genesis of choice modeling, we introduce the two basic workhorses in choice modeling, the logit and probit models. We use these two models as a platform from which to show how additional phenomena can be introduced, including multistage decision processes, dynamic models, and heterogeneity. After a description of some more advanced models, we close by illustrating how these models may be used to provide insight to marketing managers by discussing a number of choice modeling applications.

\section{8: Bayesian econometrics}

Bayesian econometric methods are particularly well suited for analysis of marketing data. Bayes theorem provides exact, small-sample inference within a flexible framework for assessing particular parameters and functions of parameters. We first review the basics of Bayesian analysis and examine three areas where Bayesian methods have contributed to marketing analytics - models of choice, heterogeneity, and decision theory. We conclude with a discussion of limitations and common errors in the application of Bayes theorem to marketing analytics.

\section{9: Structural models in marketing}

In this chapter, I provide brief discussions of what we mean by structural models, why we need them, the typical classes of structural models that we see being used by marketers these days, along with some examples of these models. I provide a basic discussion of structural models in the context of the marketing literature and limit myself largely to models of demand rather than models of firm behavior. 
10: Multivariate statistical analyses: cluster analysis, factor analysis, and multidimensional scaling

In this chapter I present three techniques - Cluster analysis, factor analysis, and multidimensional scaling - popular with marketing researchers and consultants because they help achieve frequently encountered marketing goals. Cluster analysis is useful in finding customer segments, factor analysis is useful for survey research, and multidimensional scaling is useful in creating perceptual maps.

\section{1: Machine learning and marketing}

Machine learning (ML) refers to the study of methods or algorithms designed to learn the underlying patterns in the data and make predictions based on these patterns. A key characteristic of ML techniques is their ability to produce accurate out-of-sample predictions. We review two popular machine-learning methods - decision trees and Support Vector Machines (SVM) in detail.

\section{2: Big data analytics}

The field of "Big Data" is vast and rapidly evolving. In this chapter, strict attention is paid to challenges that are associated with making statistical inferences from big data. We characterize big data by the four Vs (volume, velocity, variety and veracity) and discuss the computational challenges in marketing applications using big data. We review stochastic approximation, variational Bayes, and the methods for wide data models.

\section{3: Meta analysis in marketing}

This chapter discusses important methods and issues in using metaanalysis to develop a knowledge base in marketing. After defining metaanalysis and explaining its role in marketing, the author discusses various steps in a meta-analytic study, focusing both on design and statistical issues. He then presents a comprehensive tabular overview of published marketing meta-analyses in various subfields of marketing.

\section{4: Marketing optimization methods}

We survey the methods, advances, and insights from research and applications pertaining to Marketing Optimization Methods over the past 70 years. Specifically, we classify extant marketing optimization problems into two key typologies based on: (1) the number ("single" or "multiple") of "sales entities" and marketing input variables involved in the problem, and (2) the nature of the objective function (e.g., static or dynamic). We discuss the modeling and solving of optimization problems that fall under these 
typologies. In each example, we summarize the problem; the choice variables; the constraints; the sales response model; the objective function; the solution approach/technique; and optimization insights/principles from the solution.

\section{5: Industry applications of conjoint analysis}

This chapter reviews four applications to provide the unique flavor and demonstrate the versatility of the conjoint analysis method. The following applications are discussed: store location selection, bidding for contracts, evaluating the market value of a change in a product attribute (MVAI), push marketing strategy in a B2B context, and choice of a distribution channel.

16: How time series econometrics helped Inofec quantify online and offline funnel progression and reallocate marketing budgets for higher profits

In order to better allocate its limited marketing resources, Inofec, a small and medium entreprise (SME) in the business-to-business sector, needed to quantify how its marketing actions drive offline and online funnel progression. We developed a conceptual framework and econometric time-series model and found evidence of many cross-channel effects, in particular offline marketing effects on online funnel metrics and online funnel metrics on offline purchases. Moreover, marketing communication activities directly affected both early and later purchase funnel stages (website visits, online and offline information and quote requests). Finally, we found that online customer-initiated contacts had substantially higher profit impact than offline firm-initiated contacts. Shifting marketing budgets towards these activities in a field experiment yielded net profit increases 14 times larger than those for the status-quo allocation.

17: Panel data models for evaluating the effectiveness of direct-tophysician pharmaceutical marketing activities

We illustrate the application of dynamic panel data methods using the direct-to-physician (DTP) pharmaceutical promotions data described in an article by Mizik and Jacobson (2004). Specifically, we focus on using panel data methods to determine appropriate model specification and to demonstrate how dramatically the estimates of the DTP effectiveness change across various common model (mis)-specifications.

18: A nested logit model for product and transaction-type choice planning automakers' pricing and promotions

We develop a consumer response model to evaluate and plan pricing and promotions in durable good markets. We discuss its implementation 
in the US automotive industry, which "spends" about $\$ 50$ billion each year in price promotions. The approach is based on a random effects multinomial nested logit model of product and transaction-type choice. Consumers differ in their overall price sensitivity as well as in their relative sensitivity to alternative pricing instruments which has to be taken into account to design effective pricing programs. We estimate the model using Hierarchical Bayes methods to capture response heterogeneity at the local market level. We illustrate the model through an empirical application to a sample of data drawn from J.D. Power transaction records.

19: Visualizing asymmetric competitive market structure in large markets

Visualizing competitive relationships in large markets (i.e., markets containing over 1,000 products) is challenging. We discuss a new model called DRMABS (Decomposition and Re-assembly of MArkets By Segmentation) for such applications. DRMABS combines methods from multiple research disciplines such as biology, physics, computer science, and sociology with a new method of submarket-centric mapping to visualize asymmetric competition in large markets in a single two-dimensional map.

\section{0: User profiling in display advertising}

Constructing behavioral profiles from consumer online browsing activities is challenging: first, individual consumer-level records are massive and call for scalable high performance processing algorithms; second, advertising networks only observe consumer's browsing activities on the sites participating in the network, potentially missing site categories not covered by the network. The latter issue can lead to a biased view of the consumer's profile and to suboptimal advertising targeting. We present a method that augments individual-level ad network data with anonymized thirdparty data to improve consumer profile recovery and correct for potential biases. The approach is scalable and easily parallelized, improving almost linearly in the number of CPUs. Using economic simulation, we illustrate the potential gains the proposed model may offer to a firm when used in individual-level targeting of display ads.

\section{1: Dynamic optimization for marketing budget allocation at Bayer}

We present an Excel-based decision-support model that allows determining near-optimal marketing budgets and represents an innovative and feasible solution to the dynamic marketing allocation budget problem for multi-product, multi-country firms. The model accounts for marketing dynamics and a product's growth potential as well as for trade-offs with respect to marketing effectiveness and profit contribution. It was success- 
fully implemented at Bayer, one of the world's largest pharmaceutical and chemical firms. The profit improvement potential in this company was more than 50 percent and worth nearly $€ 500$ million in incremental discounted cash flows.

\section{2: Consumer (mis)behavior and public policy intervention}

Consumers often "misbehave." They save and exercise too little; they spend, eat, and drink too much and take on too much debt; they work too hard (or too little); they smoke, take drugs, have unprotected sex, and carelessly expose their private lives on social media. These misbehaviors, often characterized as time-inconsistent choices, may entail large costs not only to the individuals concerned, but also to society as a whole. In this chapter, I discuss how policy makers can take a theory-guided experimental approach, complemented by field data, to demonstrate consumer precommitment both as a revealed preference-based criterion for evaluating the need for policy intervention and as a tool for allowing consumers to limit their misbehaviors without imposing constraints on market participants' freedom of choice.

23: Nudging healthy choices with the 4Ps framework for behavior change

In this chapter, we share the 4Ps Framework for Behavior Change, designed to organize research findings to make them more easily applicable in the real world. We offer levers the well-meaning planner can employ to support the healthy intentions of others, and share examples of how the 4Ps Framework is being applied at Google. Although our examples focus on nudging people toward healthy food choices, similar strategies can be used to nudge people's behavior in any direction that supports their own intentions. We offer advice for influence one-time decisions via (1) the combination of choices offered, (2) the choice environment, and (3) communication about the choices. We also offer advice on supporting individuals in the development of good habits, to make better choices in any time or place.

24: Field experimentation: promoting environmentally friendly consumer behavior

This chapter discusses the challenges and rewards of conducting field experiments by sharing the details that went into conducting several large-scale field experiments within hotels. In discussion of the studies, we document three stages of conducting field experiments with outside organizations. The first stage is devoted to advice on outreach, including communication strategies to reach potential organizations. The second stage refers to securing buy-in from key stakeholders and organization partners. Lastly, we detail methodological advice in the implementation stage by highlighting potential concerns and safeguards. 


\section{5: Regulation and online advertising markets}

Online advertising has grown rapidly in recent years. The rise of this new form of advertising has generated a number of policy questions around privacy, the ability of local governments to regulate information, and antitrust in online markets. This chapter reviews three studies using a combination of field experiments and quasi-experimental variation to answer policy questions related to online advertising.

26: Measuring the long-term effects of public policy: the case of narcotics use and property crime

This chapter uses multivariate time-series methods to study one of the most serious public policy problems, the fight against narcotics abuse. The effects of methadone treatment and legal supervision of narcotics use and criminal activities were assessed by applying cointegration and error correction methods that disentangle the long-term (permanent) and the short-term (temporary) effects of intervention. Overall, the system dynamics among these variables were characterized by long-term rather than short-term relationships. Methadone maintenance treatment demonstrated long-term benefits by reducing narcotics use and criminal activities. Legal supervision, on the other hand, did not reduce either narcotics use or property crime in the long run. The chapter explores the policy implications of these findings.

\section{7: Applying structural models in a public policy context}

We present an illustration of how marketing and structural models can be applied in a public policy context. We describe the demand model in Albuquerque and Bronnenberg (2012) to evaluate the impact of the 2009 federal policy measure known as the "Car Allowance Rebate System" program (or "Cash for Clunkers") on prices and demand in the auto sector.

28: Avoiding bias: ensuring validity and admissibility of survey evidence in litigations

Despite the wide scope for survey evidence used in litigation, the relevance and usefulness of expert-submitted surveys in any legal context is dependent on how they are designed and implemented. The avoidance of bias in survey evidence is central to a survey's admissibility and the probative weight accorded to the survey expert's testimony. This chapter discusses possible sources of bias and describes methods and techniques that a survey expert can use to minimize this bias. 


\section{9: Experiments in litigation}

Often litigation outcomes hinge on very specific questions of consumer behavior (e.g., how consumers interpret a specific advertisement). Randomized experiments are instrumental in these contexts. Courts use the same criteria as academics to judge these experiments: construct, internal, and external validity. However, they place different emphases on them. For example, external validity is much more crucial in a courtroom than in an academic setting. This article discusses the similarities and differences between experiments conducted in academic social science and litigation. Furthermore, it points to a potential of the courtroom to inform academic social science that has heretofore gone unexplored.

\section{0: Conjoint analysis in litigation}

We discuss the use of consumer surveys to evaluate consumer confusion in a trademark infringement case. Because trademark owners are often unable to provide evidence of actual confusion, consumer surveys can be used to evaluate the likelihood of consumer confusion over similarity of trademarks or products. We summarize the role surveys play in trademark infringement cases and discuss their use in a trademark infringement case involving artesian bottled water from the Republic of Fiji.

\section{1: Conjoint analysis: applications in antitrust litigation}

This chapter describes an application of consumer surveys in the litigation context. This particular application of a survey differs from the typical use of market research conducted for new product development, consumer satisfaction studies, or the assessment of consumers' willingness-to-pay for a good or service. We describe and explain why and how a survey can be an important means for either Plaintiffs or Defendants to present evidence on the interpretation of a claim (here, a so-called All Natural claim displayed on the packaging of Ben \& Jerry's ice cream), as well as to evaluate the role that such a claim can play in the consumer's decision-making process.

\section{2: Feature valuation using equilibrium conjoint analysis}

This chapter discusses the use of conjoint analysis in litigation. The author summarizes key court decisions and motivates the use of conjoint analysis as a method of proof in specific litigation settings. The chapter then describes the basic elements of conjoint analysis and addresses several tactical considerations in using conjoint analysis. The specific use of conjoint analysis in a variety of litigation contexts is then summarized, including an extended summary of the use of conjoint analysis in a landmark smartphone dispute. 
33: Regression analysis to evaluate harm in a breach of contract case: the Citri-Lite Company, Inc., Plaintiff v. Cott Beverages, Inc., Defendant

We outline some basic considerations and implementation strategies regarding the use of consumer surveys and conjoint analysis in the context of complex litigation. We also describe two applications of these techniques in antitrust disputes in the payment card and infant formula supplements industries.

34: Consumer surveys in trademark infringement litigation: FIJI vs. VITI case study

Feature valuation is an important element of the marketing analytics toolkit and one of the primary motivations behind the popularity of conjoint analysis. We call attention to an important deficiency in current, consumer-centric, approaches. Surveys used for feature valuation need to include a reasonable competitive set. We demonstrate that equilibrium calculations are both necessary and feasible.

35: Survey evidence to evaluate a marketing claim: Skye Astiana, Plaintiff v. Ben \& Jerry's Homemade, Inc., Defendant

We discuss the use of regression analysis to evaluate harm in a breach of contract case involving allegations that the licensor of a product failed to use commercially reasonable efforts to promote and sell the product. Regression analysis has been widely used and accepted by US courts across a large variety of different types of cases, including labor discrimination cases, antitrust cases, and intellectual property cases. In cases involving marketing issues, regression analysis is frequently used to determine the effect of promotion on sales.

\section{6: Machine learning in litigation}

Litigation presents significant challenges involving the identification, sorting, and analysis of large amounts of data. Machine learning, which utilizes algorithms and systems that improve their performance with experience to classify information and to make predictions, is well-suited to these tasks. In this chapter, we discuss current machine learning applications in legal practice, as well as some potential applications of these techniques in support of expert witness testimony in commercial litigation. 